\title{
Research on the Control Strategy of Passive Torque Servo System for Loading at High Frequency
}

\author{
Zhisheng Ni and Mingyan Wang \\ School of Electrical Engineering and automation \\ Harbin Institute of Technology \\ awillasun@126.com
}

\begin{abstract}
In view of the problem of difficult to suppress surplus torque and to obtain high servo accuracy at high frequency in the passive torque servo system(PTSS), the neural network PID control strategy with surplus torque compensation based on dynamic fuzzy neural network (DFNN) and double-stator motor is proposed. Firstly the model of the PTSS is built and the mathematical model of surplus torque is derived. Then the model of surplus torque is identified by DFNN. The surplus torque is estimated in real time and converted directly to the control signals of outer stator of double-stator motor which produces torque to compensate the surplus torque. Finally the parameters of the neural network PID (NNPID) controller are adjusted in real time according to the Jacobian information and system error. The Jacobian information is obtained from the online identification of PTSS by RBF neural network. The simulation results show that surplus torque model is close to the actual surplus torque system, that compensation control largely eliminates the surplus torque, that system performance is improved and that the control strategy is successful.
\end{abstract}

Keywords: DFNN, double-stator motor, NNPID, surplus torque, PTSS

\section{Introduction}

The passive torque servo system (PTSS) is important equipment in the hardware-inthe-loop simulation of aircraft control system. It is mainly used in servo system and is often used to simulate the aerodynamic torque loading on the actuators in the flight. With the improvement of requirements for agility and rapidity of aircraft, response quickness, accuracy and robustness of the PTSS is required higher and higher.

Surplus torque seriously affects the dynamic performance of the PTSS. How to eliminate and suppress the influence of the surplus torque became the most important technical problems of the PTSS. The traditional methods are mostly compensation control based on structure invariance principle, Hybrid control and $\mathrm{H}^{\infty}$ control, etc. [1$3]$. But the PTSS is very strong nonlinear which makes the traditional control method difficult to obtain ideal control accuracy.

In recent years, people turn to the direction of the intelligent control. People solve the nonlinear control problem of the PTSS with fuzzy control and neural network control which has strong nonlinear processing capabilities. Learning control algorithm is proposed to improve loading precision and response speed in paper [4]. In paper [5] and [6], the neural network control is applied to electric load simulation system for 
eliminating the influence of the surplus torque. Paper [7] proposes a neural network control algorithm based on the Cerebellum Model Articulation Controller (CMAC) in the PTSS. But when these methods are applied to torque loading at the high frequency, the control effect is not ideal.

This paper designed a new method to eliminate surplus torque of PTSS through the identification of surplus torque model and double-stator compensation control. The surplus torque model is identified by DFNN. The double-stator motor whose inner stator produces torque to track the desired torque and outer stator produces torque to compensate the surplus torque is used as load motor. System adopts the neural network PID control strategy to achieve good control accuracy.

\section{PTSS Structure}

Electric load simulator is a typical PTSS with position disturbance, consisting of loading motor, PWM drives, sensors and controllers.

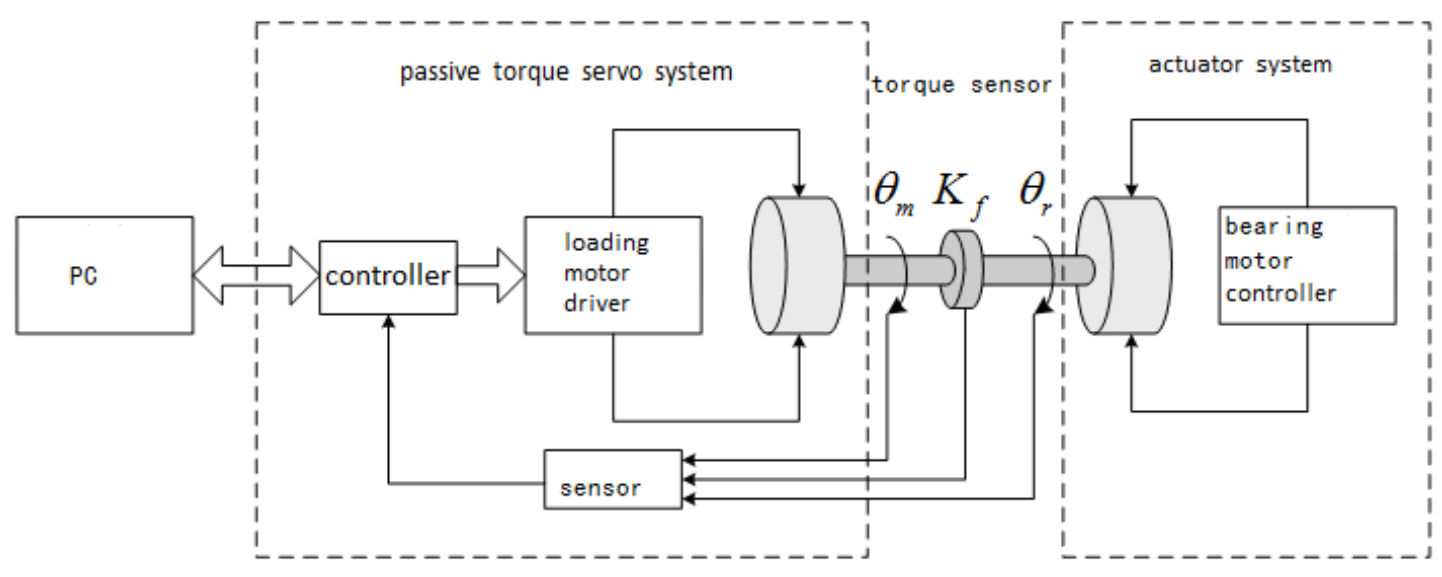

Figure 1. PTSS Structure

As shown in Figure 1, bearing motor (servos) as the loaded object connects the loading motor directly with rigid shaft. In the loading process, signals of torque and angle sensor go through the converter and enter the controller. In accordance with the simulation computer instruction, the controller outputs signals which drive the PWM driver to control output torque of the loading motor.

Double-stator permanent magnet brushless DC motor is used as the loading motor. The two stator windings could be independently controlled to load and compensate torque [9]. 


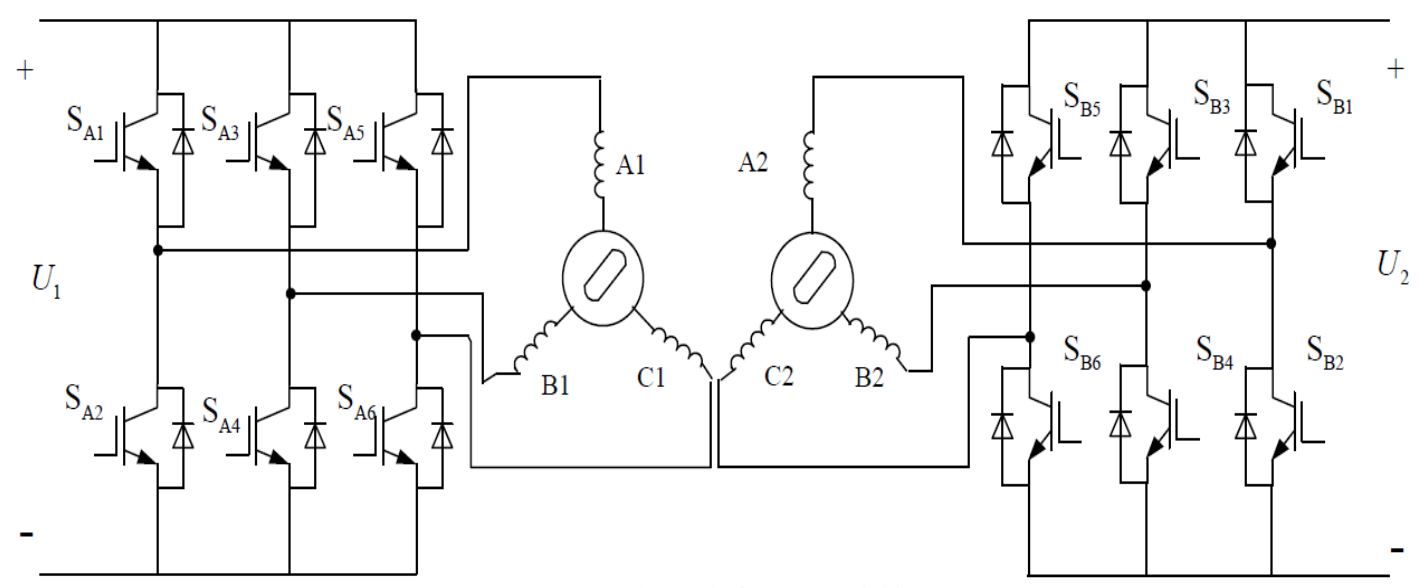

Figure 3. Double-stator Motor Drive Structure

From [10], we can obtain the load torque output of PTSS which can be described as

$$
T_{L}(s)=\frac{K_{T} K_{f} U_{m}(s)-\left(L_{m} J_{m} s^{3}+R_{m} J_{m} s^{2}+K_{e} K_{T} s\right) K_{f} \theta_{r}(s)}{L_{m} J_{m} s^{3}+R_{m} J_{m} s^{2}+\left(K_{e} K_{T}+L_{m} K_{f}\right) s+R_{m} K_{f}}
$$

Here $K_{T}$ is motor torque coefficient, $K_{f}$ is torque sensor coefficient, $L_{m}$ is inductance coefficient, $R_{m}$ is armature resistance, $J_{m}$ is the rotational inertia of motor, $K_{e}$ is the back emf coefficient, $U_{m}(s)$ is armature voltage, $\theta_{r}(s)$ is angular position of servos, $T_{L}(s)$ is torque output of PTSS.

There is no clear definition for surplus torque. In general, surplus torque is the torque output which is produced because of the movement of servos when given torque is zero. So when $U_{m}(s)=0, T_{s}(s)=T_{L}(s)$.Here $T_{s}(s)$ is surplus torque. From (1), we get the transfer function from surplus torque to angular position as

$$
\frac{T_{s}(s)}{\theta_{r}(s)}=\frac{-s\left(L_{m} J_{m} s^{2}+R_{m} J_{m} s+K_{e} K_{T}\right) K_{f}}{L_{m} J_{m} s^{3}+R_{m} J_{m} s^{2}+\left(K_{e} K_{T}+L_{m} K_{f}\right) s+R_{m} K_{f}}
$$

From (2), we can see that surplus torque is related to the angular velocity, acceleration and jerk of servos. The surplus torque has nothing to do with the control of loading motor. This provides the basis for the surplus torque compensation. 


\section{Surplus Torque Model Identification Based on DFNN}

From (2), surplus torque can be written as

$$
\begin{aligned}
& T_{s}(s)=-\frac{1}{R_{m} K_{f}}\left[\left(K_{e} K_{T}+L_{m} K_{f}\right) \mathcal{T}_{s}^{\&}(s)+R_{m} J_{m}(s)+L_{m} J_{m}(s)\right] \\
& -\frac{K_{f}}{R_{m} K_{f}}\left[K_{e} K_{T} \Theta_{r}(s)+R_{m} J_{m} \psi_{r}(s)+L_{m} J_{m}(s)\right]
\end{aligned}
$$

It can be seen from (3) that surplus torque system is a third-order dynamic system. It can be described by NARMAX model as

$$
y(t)=f\left[y(t-1), \ldots, y\left(t-n_{y}\right)\right]+g\left[x(t-1), \ldots, x\left(t-n_{x}\right)\right]
$$

Here $n_{y}=3, n_{x}=3$. The surplus torque system can be seen as six-input single-output dynamic systems. We use DFNN to identify the surplus torque model.

The structure of the DFNN is depicted in Figure 4. It is equivalent to a TSK model-based fuzzy system [11].

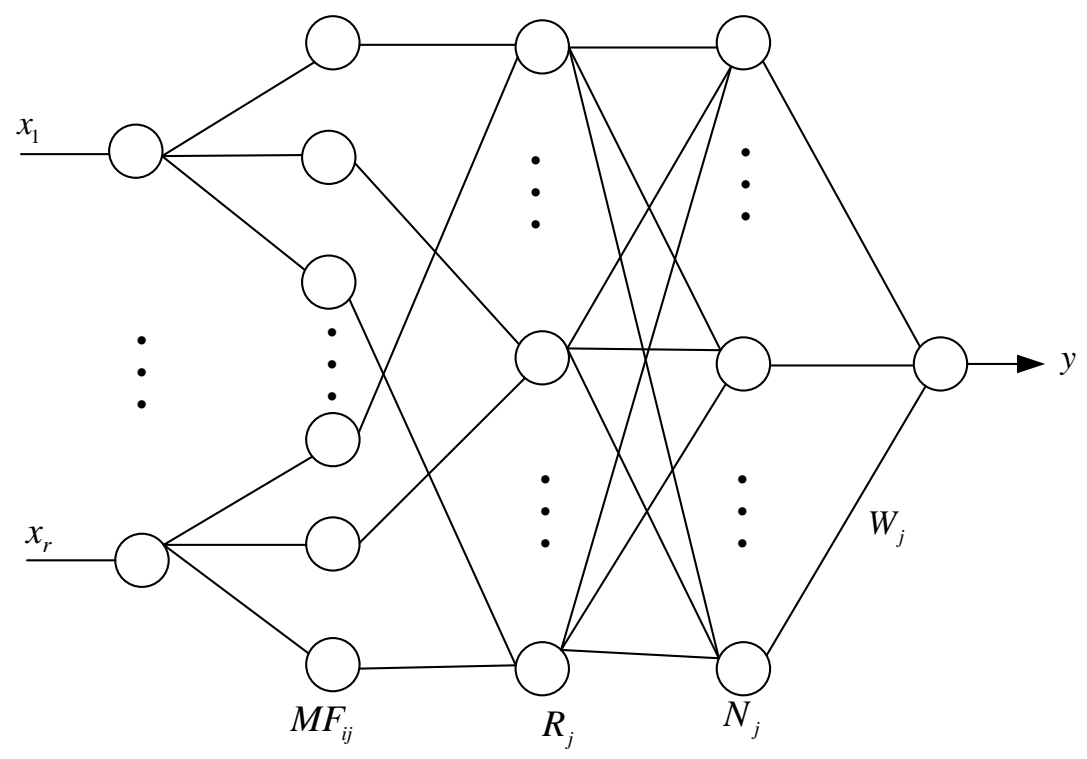

Figure 4. the structure of the DFNN

The learning algorithm is a hierarchical on-line self-organizing algorithm. Both the parameter and structure identification can be achieved quickly without initialization of parameters and structure. Algorithm flowchart is shown in Figure 5. 


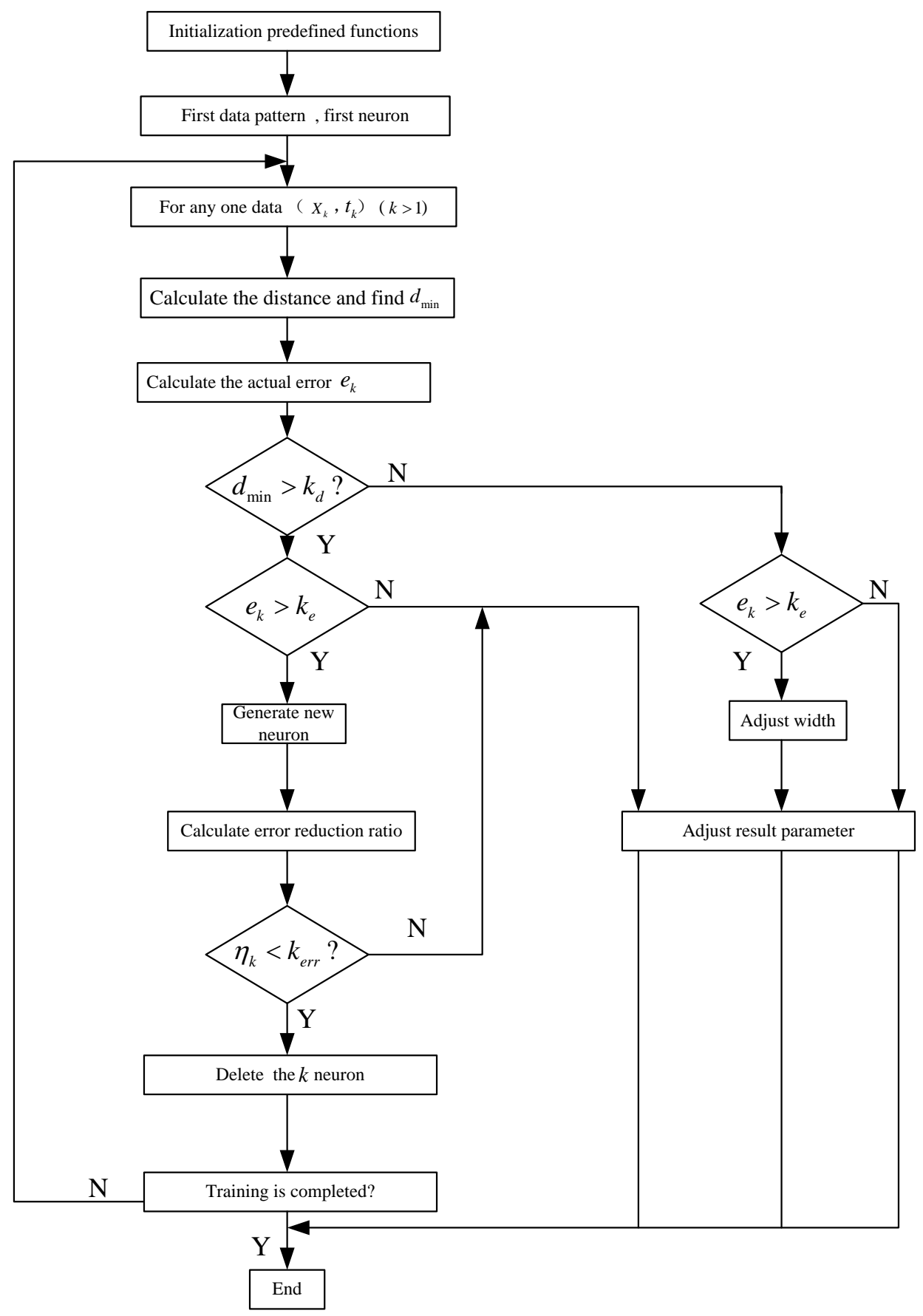

Figure 5. Algorithm Flowchart

Here, the value $k_{e}$ is chosen $a$ priori according to the desired accuracy of the D-FNN. If $\left\|e_{i}\right\|>k_{e}$, an $\mathrm{RBF}$ unit should be considered; $k_{d}$ is the effective radius of the accommodation boundary. If $d_{\min }>k_{d}$, an RBF unit should be considered; $\eta_{i}$ represents the 
significance of the $i$ th RBF unit. If $\eta_{i}<k_{e r r}$, the $i$ th RBF unit is deleted, where $k_{e r r}$ is a preset threshold.

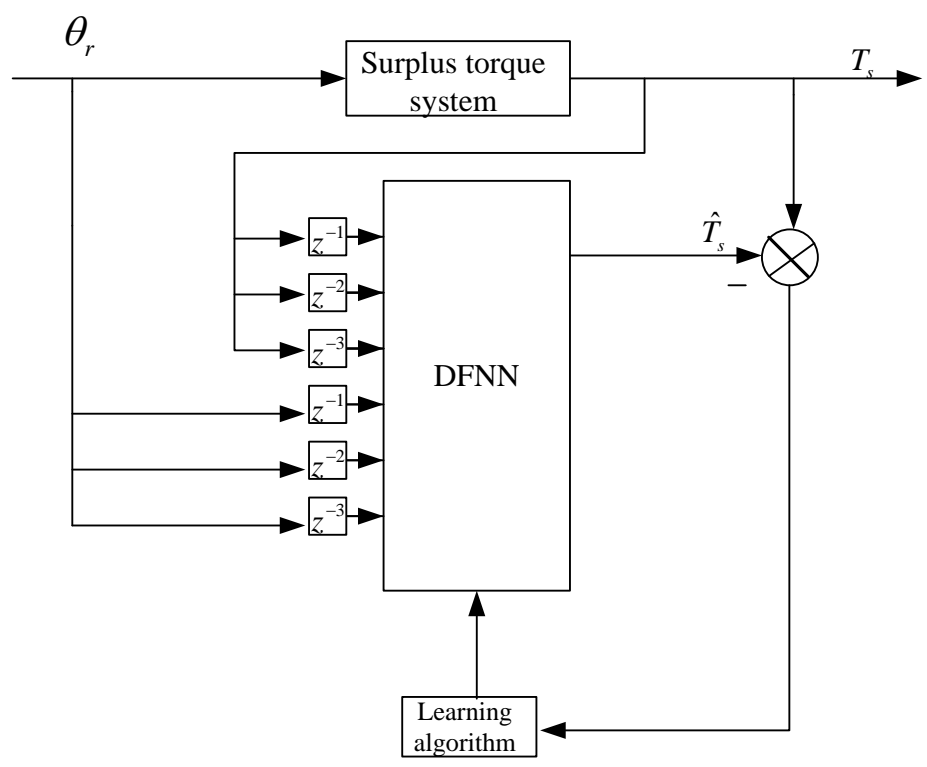

Figure 6. Diagram of Surplus Torque Identification

As shown in Figure 6, a variety of typical data are selected as training data as so the identification model is close to the actual system. In the paper, sinusoidal loading is taken as an example. Four different amplitudes and different frequencies of the sine function are selected as the input. The input and actual output became sample data. The result of identification is Figure 7 with 388 pairs as training data and 388 pairs as the test data.
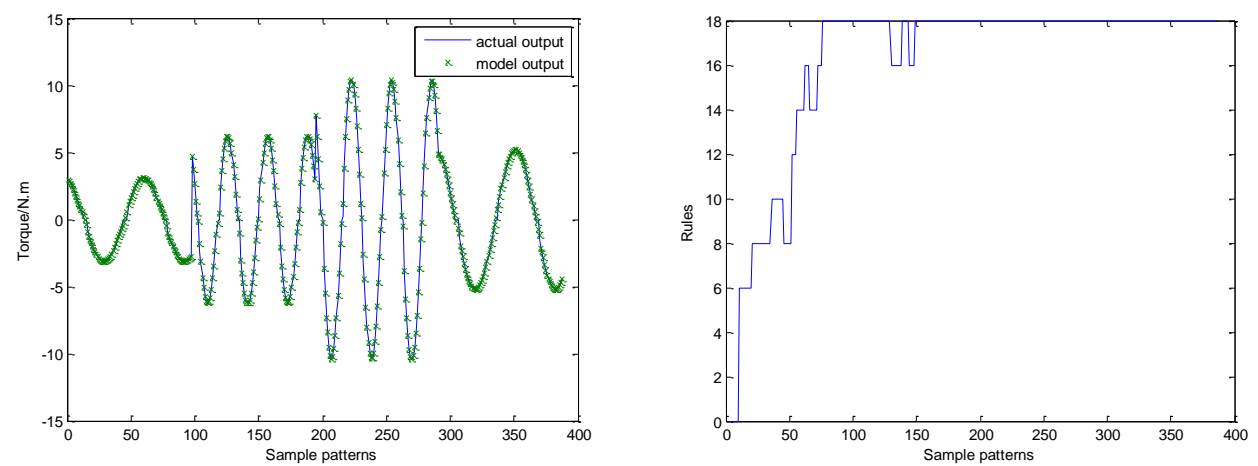

(a) Comparison between model output; (b) the number of rules in identification and actual output

Figure 7. Results of Identification

The number of rules is 18. The Root Mean Square Error (RMSE) is 0.0162 . 


\section{System Control Strategy}

Taking into account nonlinear factors of the friction and characteristics of high-frequency loading, we adopt a neural network PID control strategy with high robustness and fast response [12].

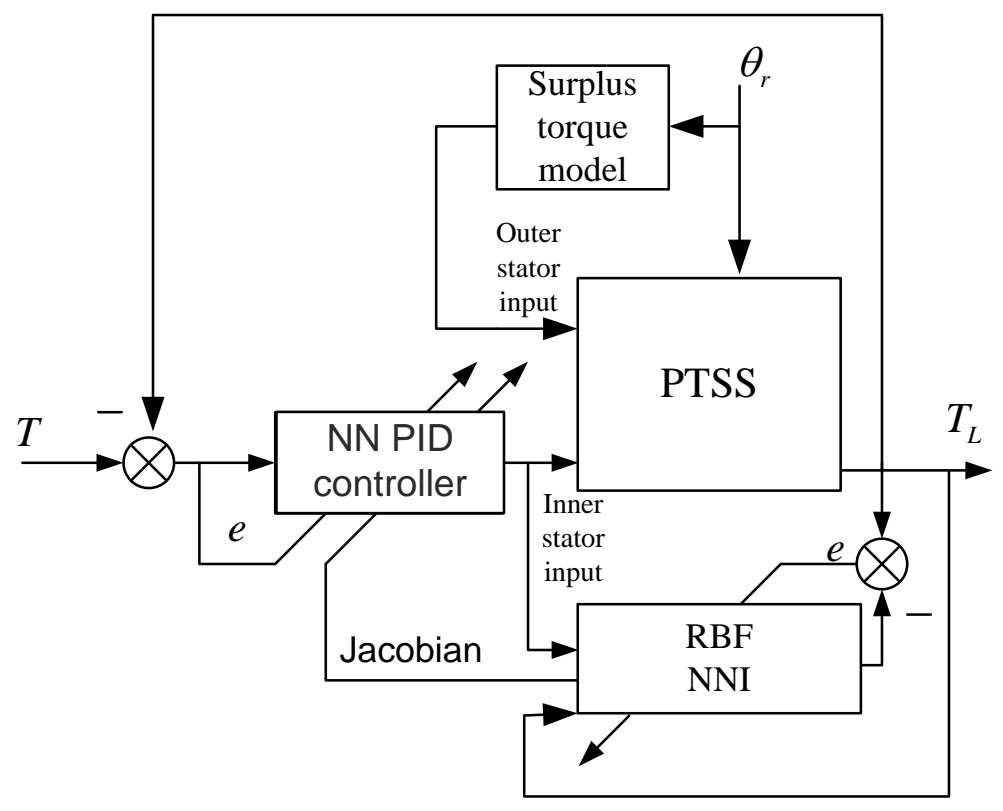

Figure 8. System Control Strategy

In Figure 8, the input and output of PTSS are the input of RBF neural network identification (NNI). The network parameters of RBF are adjusted in real time based on the identification error. NNPID controller is a single neuron PID controller which adjusts the parameters of PID based on the error of system and Jacobian matrix output by RBF NNI. The surplus torque is estimated in real time and converted directly to the control signals of outer stator of double-stator motor which produces torque to compensate the surplus torque. The inner stator is looked as loading stator which is used to track the desired torque.

\section{Simulation Results}

\subsection{Model validation}

To validate the model identified by DFNN, we set torque $T=0, \theta_{r}(t)=15 \sin 2 \pi * 10 * t$ and control system open-loop. Simulation results are shown in Figure 9. 


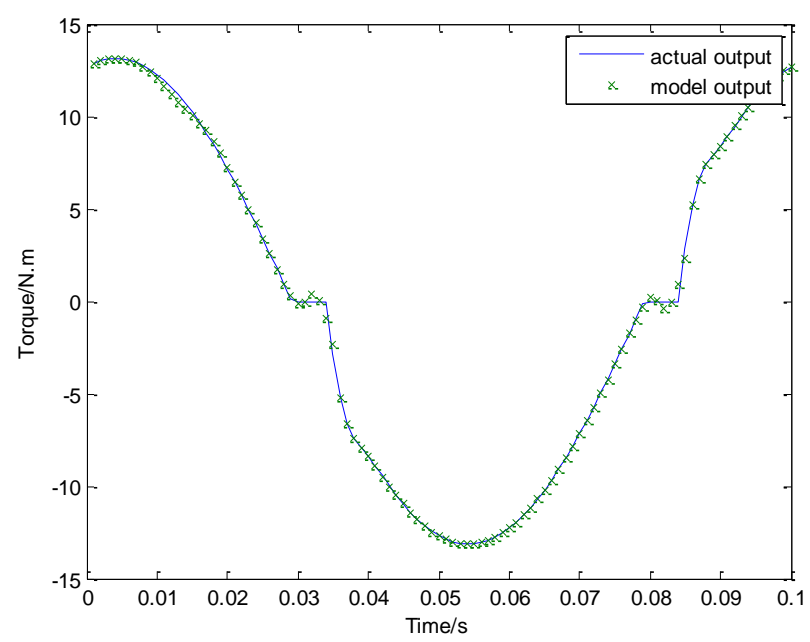

Figure 9. Comparison between Model Output and Actual Output

The generated surplus torque is $13 \mathrm{~N}$.m. The surplus torque error between the actual system and model is very small. This shows that identification of the model is very close to the real system.

\subsection{Surplus torque suppression}

To validate surplus torque suppression by double-stator, we set torque $T=0$, $\theta_{r}(t)=15 \sin 2 \pi^{*} 10 * t$ and control system closed-loop. Simulation results are shown in Figure 10.

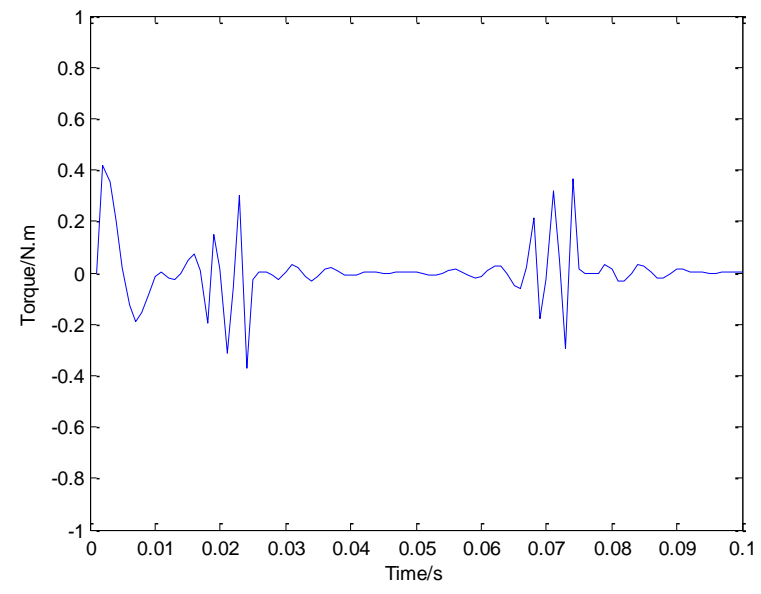

Figure 10. Surplus Torque Suppression

The max of generated surplus torque is $0.42 \mathrm{~N} . \mathrm{m}$, down by $96.7 \%$. This shows that the suppression effect is obvious. 


\subsection{System Torque tracking response}

System Torque tracking response is shown in Figure 11, where $T=50 \sin (2 \pi * 10 * t)$ and $\theta_{r}(t)=15 \sin (2 \pi * 10 * t)$.

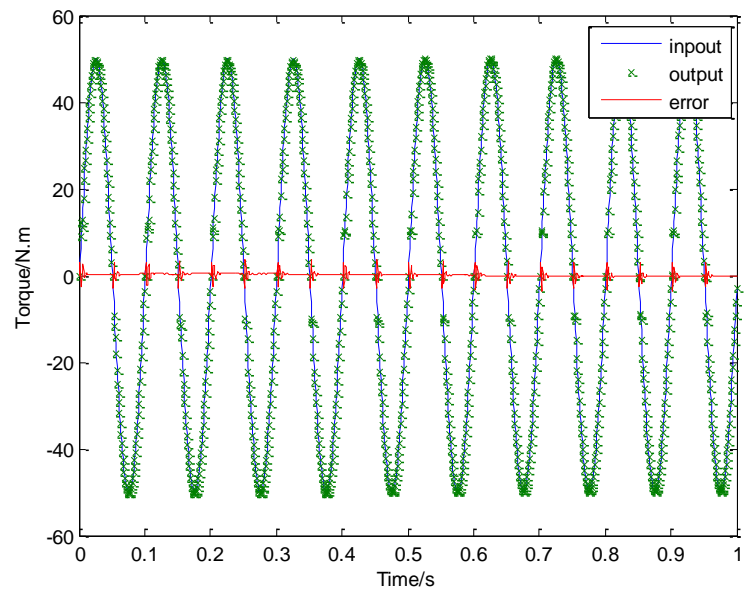

Figure 11. System Torque Tracking Response

As we can seen from Figure 11 that the phase difference is less than 1 degree between the input toque and the output torque and the magnitude error between input torque and output torque is also very small which is below $4 \%$ of the input. This shows that the system loading at $10 \mathrm{~Hz}$ is successful and the control strategy is successful.

\section{Conclusion}

The surplus torque model identified by DFNN is very close to the actual system. The method that outer stator is used directly to compensate surplus torque helps to eliminate the effects of the surplus torque on the system and maintain the stability of high-frequency system. NNPID controller which adjusted the parameters in real time according to the Jacobian information and system error ensures the stability, rapidity and precision of servo system. Simulation shows that compensation control largely eliminates the surplus torque, that system performance is improved and that the control strategy is successful.

\section{Acknowledgements}

The authors would like to appreciate the support of National Natural Science Foundation of China (51077025).

\section{References}

[1] F. Qiang, "Research on design method for passive torque servo control system and its application", Harbin Institute of Technology, (2006), pp.71-73.

[2] L. Guo and Z. J Weng, "Research on Electric Load System by Double-loop Control", Computer Simulation, vol. 28, no. 3, (2011), pp. 52-53.

[3] H. Yong and S. Li, "Research on Synchronous Control for Electric Load Simulator", Journal of Projectiles, Rockets, Missiles and Guidance, vol. 30, no. 2, (2010), pp. 42-44. 
[4] D. Feng and M. C. Chu, "Electro-hydraulics servo loading system based on learning control", Journal of Xi' an Institute of Technology, vol. 22, no. 4, (2002), pp. 46-50.

[5] Zhangjian and M. Y. Wang, "RBF Neural Network Control Strategy for Dynamic Load Simulator", Techniques of Automation \& Applications, vol. 25, no. 9, (2006), pp. 25-29.

[6] Q. Hua and Z. X. Jiao, "RBF Network Control on Hydraulic Load Simulator", Proceedings of 5th International Conference on Fluid Power Transmission and Control, (2001) April 03-05, pp. 350-354.

[7] Z. M. Ye, "Application of an Improved Arithmetic Based on CMAC in Electrical Load Simulator", Electronics Optics \& Control, vol. 18, no. 4, (2011), pp. 72-76.

[8] E. Bomme, A. Foggia and T. Chevalier, "Double air-gaps permanent magnets synchronous motors analysis", Electrical Machines Conference, (2008), pp. 1-5.

[9] W. Xiaojun, "Research on double-stator permanent magnet motor control strategy for load simulation system”, Harbin Institute of Technology, (2011), pp. 12-29.

[10] N. Zhisheng and W. M. Yan, "A novel method for restraining the redundancy torque based on DFNN", Journal of Harbin Institute of Technology, vol. 44, no. 10, (2012), pp. 47-49.

[11]M. J. Er and S. Q. Wu, "A fast learning algorithm for parsimonious fuzzy neural systems”, Fuzzy Sets System, vol. 126, no. 3, (2002), pp. 61-75.

[12] Z. Yan, "The analysis and simulation of MRAC system based on RBF network identification", Southwest Jiaotong University, (2010), pp. 4-55. 